\title{
La invisibilidad y la identidad cultural en los estudiantes indígenas de la Amazonía
}

\section{Lulaynin kikinkay manalikapaniyukaa Amasunyap ćhalakunap yaćhapakukunakaakap}

Kara te ingonijatokote aike ora onintagani agenganeegi inintaiganakero sangenaigatsi matsigengas pañaigacha anchatosigitetsarankagitekë

\section{Okantakoyetari ikantayeta atiripe sabikayetatsiri anampiki Amazoniaki ishitoriayetatsiri}

Recibido: 31 Julio 2019 Aprobado: 22 diciembre 2019

Wendy Nelly Bada Laura, Nacionalidad: Peruana Universidad Nacional Intercultural de la Amazonia, Correo: wbadal@unia.edu.pe.; ORCID: https://orcid.org/oooo-0002-0038-4601

\section{Resumen}

La identidad cultural es la base de todo desarrollo del ser humano. A través de la educación se fortalece su identidad personal y cultural, en el presente estudio se describe el nivel de visibilidad cultural de los estudiantes indígena con que llegan a la entidad formadora y la tendencia de invisibilidad cultural de los estudiantes indígenas de la Amazonía durante su formación profesional es el objetivo del presente estudio, en una muestra de 56 estudiantes indígenas Shipibo-konibo, Awajun, Yanesha, Wampis, Kichwa, Shawi, Asháninka y Achuar. El tipo de investigación fue básico, de nivel descriptivo simple, el muestreo fue no probabilístico de tipo intencional. La técnica que se utilizó la observación, el instrumento fue la ficha de observación en una escala de 1 al 5 donde uno equivale (nunca), dos equivale (casi nunca), tres equivale (a veces) cuatro equivale (casi siempre) y cinco equivale (siempre). El análisis de datos fue a través del Office Excel, teniendo como resultado significativo que la visibilidad e invisibilidad en la identidad cultural de los estudiantes indígenas de la Amazonía es de un nivel $62 \%$ y $15 \%$ respectivamente. También es notorio que cualidades esenciales que distinguen a una persona de otra cuyas raíces aún no han sido fortalecidas y que las conocen pero no las practican y la entidad formadora no incorpora contenidos propios de su cultura para recuperar y preservar su cultura e idioma.

\section{Palabras clave:}

Visibilidad, Invisibilidad, Identidad cultural, Pueblos indígenas.

\section{Lisichiku limaykuna:}

likana, mana likana, lulay kikinkay, ćhakla nunakuna.

\section{Nibarintsipage Katingaro: konijatagantsi, te ingonijate, nintiro igenganepage, timagantsipage irasi matsigenga.}

\section{Ñantsipe ayoyeteri:}

Ayotakayeteri, omanayetachari, Kantakayeri ashaninkati, atsipanampiyetari.

\section{Datos de la autora}

Wendy Nelly Bada Laura: Investigadora y docente de educación especial, especialidad en educación inicial y educación intercultural rural intercultural bilingüe. Magister en Psicología Educativa por la Universidad César Vallejo, Ciudad de Trujillo. 


\section{Invisibility and Cultural Identity in Indigenous Students From the Amazon}

\begin{abstract}
Cultural identity is the basis of all human being development. Through education their personal and cultural identity is strengthened, this study describes the level of cultural visibility of the indigenous students with whom they arrive at the training entity and the trend of cultural invisibility of the indigenous students of the Amazon during their Vocational training is the objective of this study, in a sample of 56 indigenous students Shipibo-konibo, Awajun, Yanesha, Wampis, Kichwa, Shawi, Asháninka and Achuar. The type of investigation was basic, of a simple descriptive level, the sampling was non-probabilistic of the intentional type. The observation technique was used, the instrument was the observation sheet on a scale of 1 to 5 where one equals (never), two equals (almost never), three equals (sometimes) four equals (almost always) and five It is equivalent (always). The data analysis was through Office Excel, with a significant result that the visibility and invisibility in the cultural identity of the indigenous students of the Amazon is $62 \%$ and $15 \%$ respectively. It is also notorious that essential qualities that distinguish a person from another whose roots have not yet been strengthened and who know them but do not practice them and the training entity does not incorporate contents of their culture to recover and preserve their culture and language.
\end{abstract}

\section{Keywords}

Visibility, Invisibility, Cultural identity, Indigenous peoples.

\section{Invisibilidade e identidade cultural nos estudantes indígenas da Amazônia}

\section{Resumo}

A identidade cultural é a base de todo o desenvolvimento do ser humano. Por meio da educação, fortalece-se a identidade pessoal e cultural. Este estudo descreve o nível de visibilidade cultural com que chegam os estudantes indígenas à entidade de formação e a tendência de invisibilidade cultural dos estudantes indígenas da Amazônia durante a formação profissional, esse é o objetivo deste trabalho. Em uma amostra de 56 estudantes indígenas Shipibo-konibo, Awajun, Yanesha, Wampis, Kichwa, Shawi, Asháninka e Achuar. O tipo de investigação foi básico, de nível descritivo simples, a amostragem não probabilística do tipo intencional. Utilizou-se a técnica de observação, o instrumento foi a folha de observação em uma escala de 1 a 5 , onde um é igual (nunca), dois é igual (quase nunca), três é igual (às vezes) quatro é igual a (quase sempre) e cinco é equivalente (sempre). A análise dos dados foi realizada no Office Excel, com resultado significativo de que a visibilidade e invisibilidade na identidade cultural dos estudantes indígenas da Amazônia é de 62\% e 15\%, respectivamente. Também é notório que as qualidades essenciais que distinguem uma pessoa de outra cujas raízes ainda não foram fortalecidas e que as conhecem, mas que não as praticam, e a entidade de treinamento não incorpora conteúdos de sua cultura para recuperar e preservar sua cultura e idioma..
Palavras-chave:

Visibilidade, invisibilidade, identidade cultural, povos Indígenas. 


\section{Introducción}

La visibilidad de las cuarentitrés culturas y lenguas indígenas en la Amazonía está poco difundida por parte de las autoridades; sin embargo, existe el riesgo de que se extinga y tenga que desaparecer con ello su legado cultural. Es necesario analizar la problemática. Al respecto, Hechavarría y Piclin (2012) manifiestan que ante un mundo intolerante, las políticas globalizadoras neoliberales tratan de negar y obstaculizar el desarrollo de los países del Tercer Mundo con el propósito de que olviden su herencia, tradiciones orales, manifestaciones culturales hasta olvidar su verdadera identidad. Otros autores, mencionan que la sociedad actual que discrimina, desconoce, margina al indígena, pues cualquier acción de cambio va a conducir a la aculturación, a la asimilación, a la desaparición del indígena, y será inevitable la pérdida de la identidad (Berdichewsky y Vives, 1970). Además la OEI (2008) rechaza contundentemente la crítica que a menudo se hace de la diversidad cultural como causa de conflictos. Cabe señalar que entre los intentos de invisibilidad de la diversidad cultural, cuando la homogenización es aceptar "lo propio" como lo único bueno, lo verdadero y que los otros representan "el mal", la causa de los problemas. Por ejemplo, la diversidad lingüística es vista por muchas personas como inconveniente, peligroso y como castigo divino, porque su existencia impide el entendimiento entre seres humanos. Mayor (2000) reconoce que la diversidad lingüística ha sido y sigue siendo víctima de fuertes prejuicios. Sin embargo, coincide en reconocer que los bilingües suelen poseer una maleabilidad y flexibilidad cognitiva superior a los monolingües. Podemos decir que una forma de invisibilizar la identidad cultural es a través de la educación tradicional homogénea y monocultural, porque todavía el sistema educativo no logra un cambio que vaya más allá de medidas relacionadas con nuevos modelos pedagógicos. UNICEF (2008). Recordemos que a la llegada de los españoles a América Latina, fueron los primeros genocidas y el evangelio jugó un papel importante modificando la vida religiosa de los indígenas, la prohibición de las prácticas autóctonas; se prohibió el uso de la lengua indígena, se polarizó la cultura entre el dominante y el dominado (Stavenhagen, 2001). González (2008) refiere que los pueblos indígenas demandan su derecho a la educación en su lengua, y también en su cultura. A partir de nuestra historia vale preguntarse ¿Quiénes somos en la actualidad? ¿Qué idioma debemos hablar? ¿Cuál es nuestro origen? ¿Qué educación debemos recibir? ¿Qué no conocemos? Son preguntar que son parte de nuestra identidad cultural, y desarrollo ante la pluralidad y diversidad cultural. Si se tiene en cuenta los fines para los que fue creada la Universidad Nacional Intercultural de la Amazonía (UNIA) que alberga a dieciséis pueblos indígenas de la Amazonía, es importante conocer ¿Cuál es el nivel de visibilidad e invisibilidad de la identidad cultural de sus estudiantes indígenas de la Amazonía? que tuvo como objetivo describir el nivel de visibilidad e invisibilidad de la identidad cultural de los estudiantes Shipibo-konibo, Awajun, Yanesha, Wampis, Kichua, Shawi, Ashaninka. La hipótesis fue: el nivel de visibilidad e invisibilidad influye significativamente en la identidad cultural de los estudiantes de la Amazonía, fue un estudio descriptivo comparativo entre las muestras.

\section{Marco Teórico}

UNICEF (2008) llevó a cabo una investigación comparativa entre Ecuador, Perú y Bolivia cuyo objetivo fue desarrollar y expandir el uso efectivo y culturalmente apropiado de las metodologías de enseñanza, curriculum y materiales para la educación intercultural bilingüe. Los resultados en Ecuador como en Perú no incluyen las demandas educativas de las poblaciones indígenas. La incorporación de los saberes al currículo son irrelevantes, y no son traducidos en logro de aprendizaje. Lo que se enseña pertenecen en su mayoría a la cultura hegemónica occidental. Se habla el español y no existe la comprensión y respeto a las lenguas nativas. No se 
considera el perfil del educando que sea intercultural. Conclusiones: los tres países deberían responder a las necesidades y expectativas del problema educativo de los pueblos indígenas, incorporando los saberes culturales en las diversas áreas del conocimiento.

EIBAMAZ (2008) realizó una investigación sobre los valores y antivalores de Pueblos y Nacionalidades Indígenas de la Amazonía. Tuvo como finalidad incorporar el tema de valores en el proceso de enseñanza y aprendizaje de la niñez con una pedagogía intercultural. Se realizó en 6 pueblos y nacionalidades de la Amazonía Shipibo y Ashaninka de Perú, Achuar y Kichwas de Ecuador, Chiquitano y Siriono de Bolivia. La realidad, el mundo, el universo, es diametralmente opuesta a la del occidente, donde se privilegia y se erige como valor a la independencia y a la autonomía, buscando la realización y felicidad personal. El pensamiento indígena nos dice que todo tiene vida, todos venimos y somos parte de un mismo espíritu de vida. Las relaciones de convivencia entre los seres habitan los mundos, es decir, seres humanos, plantas, animales, cuerpos celestiales se complementan, en equilibrio y armonía. Luego de haber profundizado los conocimientos y valores de los pueblos indígenas de la Amazonía, la escuela debe garantizar este conocimiento acercándose más a los valores de los pueblos indígenas, también debe convivir con los actores de la comunidad para ser una educación en la vida, en la práctica y no el discurso de introducir aprendizajes ajenos a su cultura. La educación indígena no se ha atrevido a crear otra escuela por los requisitos que exigen los modelos de estudio y sobre todo por la idea equivocada de que el modelo occidental plantea.

Por consiguiente, el concepto según Salgado (1999) de la identidad cultural, se moldea desde la edad temprana con nuestras costumbres, hábitos, fiestas, bailes, modos de vida, todo aquello que forma parte de nuestro folklore y que es expresión misma de nuestro pasado y presente con proyección al futuro. Por otro lado, Batzin (citado por Grimaldo, 2006) define la identidad cultural a la manera en la cual un pueblo se autodefine. Para Gorosito (1998) la identidad es un aspecto de la reproducción cultural; es la cultura internalizada en sujetos y apropiada bajo la forma de una conciencia de sí, en el contexto de un campo limitado de significaciones compartidas con otros. Para Gissi (1996) es necesario tener dos nociones fundamentales: la endógena (es como el pueblo se autodefine) y exógena (como lo definen los demás). Según Erickson (1980) la identidad cultural es lograr llegar a ser un adulto único con un papel importante en la vida. Así también los sentimientos de mismidad personal, o sea el conjunto de cualidades esenciales que distinguen a una persona de otra. Son cuatro los estados de identidad étnica: difusión cultural (hace poca o ninguna exploración de su origen étnico), experimenta una crisis de identidad, exclusión (tiene sentimientos claros sobre todo actitudes que asimila en su hogar), moratoria (se siente confundido al hacer lo que esto significa) y logro de identidad (entiende y acepta su origen).

Cabe señalar que el propósito de brindar una educación intercultural en América Latina y el Caribe, responde a las necesidades de una educación diferencial, distinta a una educación tradicional homogénea y monocultural, que tenga características de una educación heterogenia y conlleve a la generación de conocimientos, reconocer que el multilingüismo promueve la unidad en la diversidad y el entendimiento internacional e impulsar y apoyar la revitalización de lenguas minoritarias (Naciones Unidas, 2014). Del mismo modo, la diversidad también atribuye a la agricultura, la gastronomía, la tradición oral, la música, las artes visuales, la arquitectura, que son parte de la riqueza de la humanidad. Señala Sen y Kliksberg (2007) que la armonía es la esperanza del mundo contemporáneo porque radica en la pluralidad de nuestras identidades. Además, toda persona debe tener la posibilidad de expresarse, crear y difundir en su lengua materna; toda persona tiene derecho a una educación y una formación de calidad 
que respeten plenamente su identidad cultural... (Pacto Internacional de Derechos Económicos, Sociales y Culturales. 1976, en sus Artículos 13 y 15). Los pueblos indígenas tienen derecho a revitalizar, utilizar, fomentar y transmitir a las generaciones futuras sus historias, idioma, tradiciones orales, filosofías, sistemas de escritura y literaturas, ... así como a mantenerlas. También tienen derecho a solicitar que la educación que se las imparta en su propio idioma, métodos culturales de enseñanza y aprendizaje; del mismo modo una educación debe impartir en su propia cultura (Declaración Universal de Derechos de los Pueblos Indígenas: $\operatorname{Art}^{\circ} 13$ y 14, 2006). Para responder a las necesidades e intereses de los pueblos, los programas y servicios deberán abarcar su historia, sus conocimientos y técnicas, sistema de valores, sociales, económicos y culturales. (Convenio $\mathrm{N}^{\circ} 169$ de la OIT, 2014). Acrecentar y transmitir de modo permanente la herencia científica, tecnológica, cultural y artística de la humanidad, así como también la afirmación y transmisión de la diversidad de identidades culturales del país. (Ley Universitaria $\mathrm{N}^{\circ} 30220,2014$ )

Es importante reconocer el valor e importancia de los conocimientos indígenas, tomándolos como eje de aprendizaje y formación profesional para la sociedad en su conjunto. Según el fundamento pedagógico de Paulo Freire, encaja, lógicamente, con la realidad y la necesidad de una educación diferencial dentro de la diversidad. Dussel (2002) compara la pedagogía de Piaget y Vygotsky con la psicología de Kohlberg; afirma que a pesar de sus méritos, permanecen en una concepción individualista del proceso educativo y del desarrollo del sujeto. En cambio, Freire (1992) sostiene en el centro de su teoría y práctica pedagógica que es el elemento de relación con los demás de que supone todo crecimiento personal, y el hecho de que éste se ha de dar dentro de una comunidad que también se va transformando a lo largo de la educación mutua de sus miembros, enfatiza la "cualidad relacional" del ser humano, que pasa a ocupar un lugar central en su visión del proceso educativo; valora la sabiduría de los campesinos analfabetos; dice que es importante evidenciar la sabiduría de los educandos que sale a relucir; descubrir la sabiduría del otro requiere humildad por parte del educador.

Freire (1992) afirma: No hay diálogo si no hay humildad. La pronunciación del mundo, con el cual los hombres lo recrean permanentemente, no puede ser un acto arrogante.

Freire manifiesta que la educación debe comenzar por la superación de la contradicción educador - educando. Debe fundarse en la conciliación de sus polos, de tal manera que ambos se hagan, simultáneamente, educadores y educandos. Además, Freire relaciona la educación formal como la forma de prohibir para ser feliz, un desafortunado distanciamiento entre los hombres que se relaciona con un doloroso rechazo a la vida. Enfocando su pedagogía con la propuesta de From (1992) "humanizar la vida", es decir, haciendo que respondan a las necesidades específicamente humanas, que implican el desarrollo de una equilibrada vinculación afectiva con los otros hombres y con el mundo.

La horizontalización de las relaciones humanas que propone llevar a cabo en el acto educativo, es decir, apunta a una doble transformación: en el corazón de la persona y en las estructuras sociales. Según Freire (1992) define la enajenación, en términos psicológicos, como la incorporación de algunas creencias ajenas operantes en nosotros, que simulan ser propias y favorecen al sujeto oprimido; que, así, vive engañado. La ideología sería la lógica del opresor que se incorpora al pensamiento del oprimido; aun más, la lógica de una estructura producida por una civilización creada por el hombre contra sí mismo y que oculta su enajenación. Para Jaspers (1958) toda pérdida y fallo en la comunicación es propiamente una pérdida del ser. 


\section{Metodología}

Esta es una investigación básica, de nivel descriptivo simple, de diseño no experimental descriptivo comparativo; donde $M_{1}, M_{2}, M_{3}, M_{n}$ son los estudiantes indígenas y $o_{1}, o_{2}, o_{3} \ldots o_{n}$ es la observación de la identidad cultural y $\mathrm{O}_{1}=\mathrm{O}_{2}=\mathrm{O}_{3}$ es la comparación de los resultados.

$$
\begin{array}{cc}
M_{1} & O_{1} \\
M_{2} & O_{2} \\
M_{3} & O_{3}^{3} \\
O_{1}^{=} O_{2} & O_{3}^{2}
\end{array}
$$

La población estuvo constituida por 380 estudiantes indígenas de la UNIA, de las cuatro Carrera Profesionales: Inicial Bilingüe, Primaria Bilingüe, Ingeniería Agroforestal acuícola y Ingeniería Agroindustrial perteneciente de los pueblos indígenas Shipibo-konibo, Awajun, Yanesha, Wampis, Kichwa, Shawi, Asháninka y Achuar; la muestra estuvo constituida por 56 estudiantes indígenas, el muestreo fue no probabilístico de tipo intencional. La técnica que se utilizó: la observación; el instrumento fue la ficha de observación, en una escala de 1 al 5 , donde uno es (nunca), dos (casi nunca), tres (a veces) cuatro (casi siempre) y cinco (siempre). El análisis de información se hizo a través del Windows Excel.

\section{Resultados}

Según los resultados, el nivel de identidad cultural de los estudiantes indígenas de las cuatro carreras tiene como promedio el $51 \%$ es decir, se visibiliza en un nivel (A veces) y significa que los estudiantes a veces preparan sus platos típicos, elaboran sus artesanías, se visten con traje típico para participar en ceremonias de la comunidad, se comunican en lengua materna, hablan pero no leen ni escriben, practican sus conocimientos ancestrales (chacra, bosque y agua), practican los secretos de la naturaleza para la conservación de los recursos naturales, rescatan los relatos orales y los juegos ancestrales, tal como se muestra en la figura 1.

\section{Figura 1}

\section{Nivel de visibilidad de la identidad cultural de los estudiantes de las cuatro Carreras Profesionales de la UNIA}

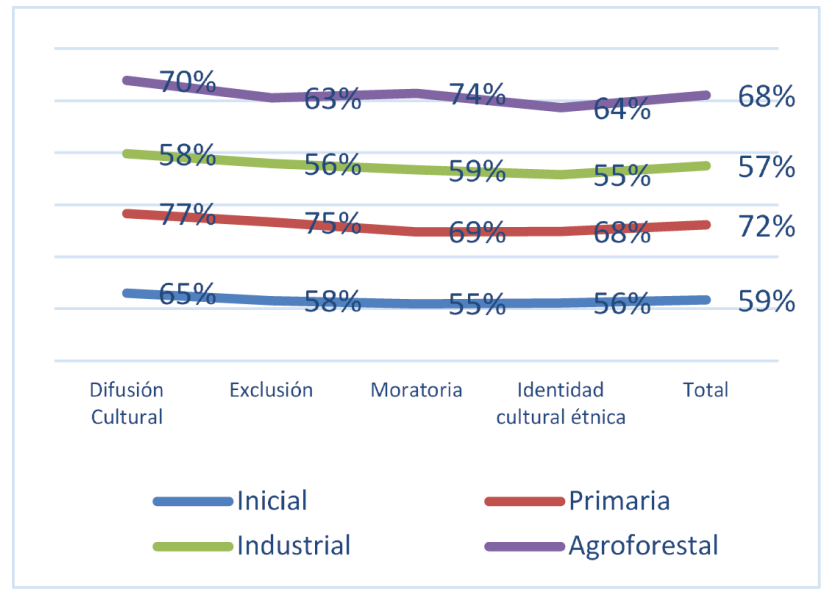

Fuente: datos de la ficha de registro. 
El promedio del nivel de visibilidad de la identidad cultural de los estudiantes indígenas por pueblos originarios de la Amazonía, es de $62 \%$ que equivale a (casi siempre). Tal como se muestra en la figura 2, se obtuvo que los estudiantes Shipibo -Konibo tienen un promedio de 50.8\% equivalente, los estudiantes Wampis obtuvieron 45\%, los estudiantes Yanesha 59.2\%, los Awajun 51\%, los Kichua 47.2\%, los Ashaninka 43\%, los Shawi 46.2\%, equivalentes a la escala (Aveces), con excepción de los estudiantes Achuar con un promedio de $38 \%$ equivalente a (Casi nunca). La identidad cultural se visibiliza en los estudiantes según las dimensiones difusión de la identidad, exclusión, moratoria e identidad cultural étnica, que significa que los conocimientos de las prácticas culturales, gastronómicas, tradiciones orales, el dominio del idioma, participación en ceremonias, secretos, arte, vestimenta, valores y principios, juegos ancestrales a un nivel de conocer, pero no del saber hacer que del ser parte de uno mismo.

\section{Figura 2}

\section{Nivel de visibilidad de la identidad cultural de los estudiantes por pueblo indígena de la Amazonía}

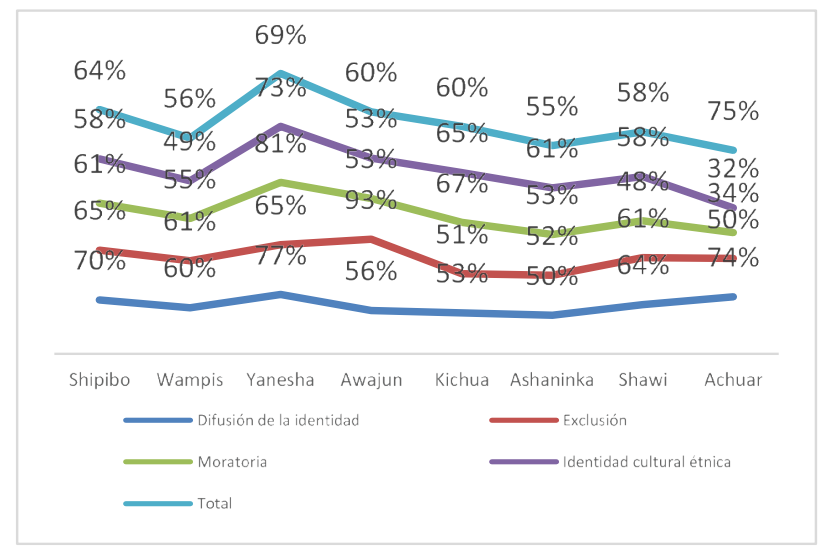

Fuente: datos de la ficha de registro

Figura 3

\section{Nivel de Invisibilidad de la identidad cultural de los estudiantes indígenas de la Amazonía}

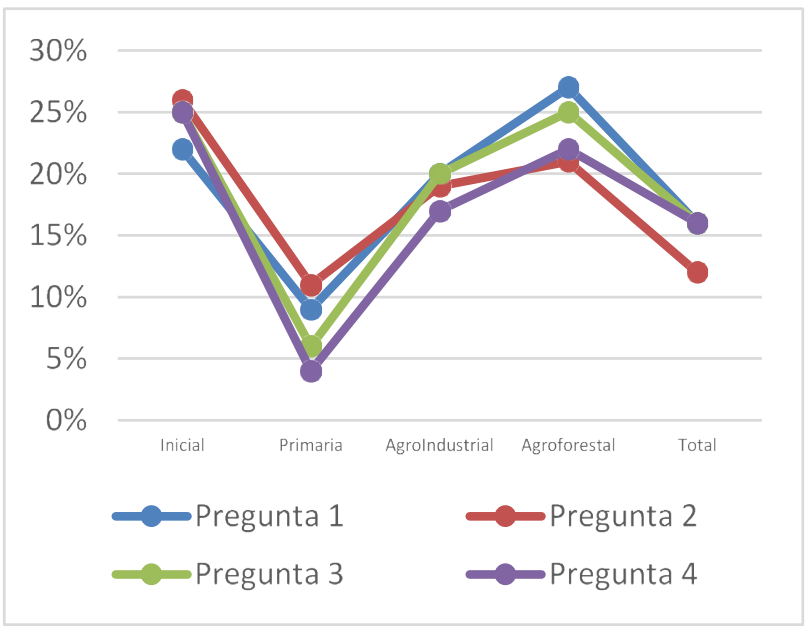

Fuente: datos de la ficha de registro 
Los resultados de la invisibilidad de la identidad cultural de los estudiantes indígenas de la Amazonía tuvieron como promedio el $15 \%$. El resultado por carrera profesional fue: Inicial Bilingüe 19.6\%, Primaria Bilingüe 6\%, Ingeniería Agrolndustrial 15\% y Agroforestal Acuícola 19\%, el que es un nivel muy bajo porque los intervalos se comprenden en la escala (Nunca). Así mismo, entre los estudiantes Shipibo -Konibo se tuvo como promedio el $17.6 \%$ equivalente, los estudiantes Wampis obtuvieron $13.4 \%$, los estudiantes Yanesha $14.4 \%$, los estudiantes Awajun 14.6\%, los estudiantes Kichwa 14.5\%, los estudiantes Ashaninka 14.5\%, los estudiantes Shawi $16 \%$ equivalente a la escala (Aveces). Los estudiantes Achuar $15.6 \%$, cuyos resultados también están en la escala de nunca; por consiguiente, la educación que reciben los estudiantes está orientada a la invisibilidad de las culturas.

\section{Discusión}

El nivel de visibilidad e invisibilidad influye significativamente en la identidad cultural de los estudiantes de la Amazonía.

Los resultados obtenidos de la visibilidad de la identidad cultural fueron en promedio $62 \%$, lo que equivale a (Casi siempre) y según el promedio de los pueblos indígenas fue $48.9 \%$ en la escala (A veces), excepto del pueblo Achuar de 38\% (Casi nunca), es decir, estos resultados guardan relación con lo que sostiene Salgado (1999) que la identidad cultural se moldea desde edad temprana con nuestras costumbres, hábitos, fiestas, bailes, modos de vida, todo aquello que forma parte de uno nuestro, Según Erickson (1980) manifiesta que la identidad cultural es lograr llegar a ser un adulto único, con cualidades esenciales que distinguen a una persona. Sin embargo, los resultados demuestran que a veces exploran su origen étnico, no tienen sentimientos claros sobre todo actitudes que asimilan en su hogar, se sienten confundidos se hace lo que esto significa, y a veces entienden y aceptan su origen.

Los resultados de la invisibilidad de la identidad cultural por carrera revelan que el promedio fue $15 \%$ lo cual equivale a (Nunca) y el promedio de los 8 pueblos indígenas de la Amazonía es $15 \%$ en la escala (Nunca), es decir, estos resultados guardan relación con lo que sostiene, EIBAMAZ (2008) que los conocimientos y valores de los pueblos indígenas son profundizados entre sus pares, pero la institución formadora debe garantizar el acercamiento más a los valores de los pueblos indígenas y adoptar estrategias para convivir con la comunidad evitar introducir aprendizajes ajenos a su cultura e implementar modelos de estudio occidentalizados; también los resultados de UNICEF (2008) refuerzan la incorporación de los saberes al currículo y que estos no son traducidos en logros de aprendizaje, pues en su mayoría pertenecen a la cultura hegemónica occidental; se habla el español y sin renunciar al uso del Castellano, no existe el dominio de leer, escribir y traducir las lenguas nativas. La entidad formadora no considera el perfil del educando que sea intercultural. Estos resultados tienen sustento teórico cuando Freire (1992) sostiene en el centro de su teoría y práctica pedagógica al elemento de relación con los demás que supone todo crecimiento personal, y el hecho de que este se ha de dar dentro de una comunidad que también se va transformando a lo largo de la educación mutua de sus miembros.

\section{Conclusiones}

El nivel de la visibilidad e invisibilidad en la identidad cultural de los estudiantes indígenas de la Amazonía alcanza al 62\% (A veces) y 15\% (Nunca) respectivamente. Se visibiliza cómo un 
conjunto de cualidades esenciales que distinguen a una persona de otra cuyas raíces aún no han sido fortalecidas, conocen pero no practican y la entidad formadora no incorpora contenidos propios de sus culturas para recuperar o preservar su cultura e idioma.

El nivel de visibilidad e invisibilidad en la difusión cultural, exclusión, moratoria e identidad cultural étnica es alto en el pueblo Awajun y Yanesha, es decir, perdura el dominio del idioma, el arte, la vestimenta, el idioma, la identificación, prácticas culturales, valores y principios, a diferencia del Shipibo -Konibo, Wampis, Kichwa, Ashaninka, Shawi y Achuar, sin embargo la entidad formadora no brinda una enseñanza en su idioma, un espacio de revalor dentro del aula, por no contar con el perfil docente adecuado a la formación intercultural. 
Referencias bibliográficas

Berdichewsky, B. y Vives, C. (1970). Para una política de acción indigenista en el área araucana, mimeografiado, Santiago-Chile: Corporación de la Reforma Agraria (Cora). 6 p.

Convenio 169 de la OIT, (2014). sobre pueblos indígenas y tribales en países independienntes.

Declaración Universal de Derechos de los Pueblos Indigenas (2006)

Dussel, E. (2002). Ética de la liberación en la edad de la globalización y de la exclusión. Madrid: Trotta.

EIBAMAZ (2008). Valores de los pueblos y nacionalidades indígenas de la Amazonía. Bolivia, Ecuador y Perú.

Erickson, E. (1980). Identidad, juventus y crisis 2. Ed. Madrid, Taurus

Freire, P. (1992). Pedagogía del oprimido. Madrid: Siglo XXI.

From, E. (1992). Psicoanalisis de la sociedad contemporánea; Madrid: FCE.

Gissi, J. (1996). Precondiciones técnicas para diagnosticar la identidad latinoamericana. En Revista Estudios Sociales Nº 87.p. 159-167.

González, E. (2008). Los profesionistas indios en la educación intercultural. Etnicidad, intermediación y escuela en territorio mixe. México: Universidad Autónoma Metropolitana, Editorial Juan Pablos.

Gorosito, A. (1998). Identidad, cultura y nacionalidad En Bayardo; Lacarrieu (Compilaciones) globalización e identidad cultural. Buenos Aires: CICUUS. Pp. 101-112.

Grimaldo, M. (2006) Identidad y Política en el Perú. Lima, Perú.

Hechavarria, I. y Piclin, J. (2012). El desarrollo de la identidad cultural mediante el estudio del patrimonio histórico y su vínculo con la historia local. Cuba

Jaspers, K. (1958). Filosofía. Revista de Occidente Ediciones de la Universidad de Puerto R.

Ley Universitaria $\mathrm{N}^{\circ} 30220$ (2014)

Mayor, F. (2000). Un Mundo Nuevo. Barcelona, UNESCO, Círculo de lectores.

Naciones Unidas (2014). Sobre los Derechos de los Pueblos Indígenas. Lima.

Organización de Estados Iberoamericanos para la Educación, La Ciencia y la Cultura OEI (2008). Diversidad Cultural. Área Cultura. Madrid. España

Pacto Internacional de Derechos Económicos, Sociales y Culturales (1976)

Salgado, C. (1999). ¿Quiénes somos los peruanos? Una perspectiva psicológica de la identidad nacional. Lima, Perú.

Sen y Kliksberg (2007). Primero la gente, Barcelona: Deusto.

Stavenhagen, R. (2001). La diversidad cultural en el desarrollo de las Américas Los pueblos indígenas y los estados nacionales en Hispanoamerica. México.

UNESCO (2001). Declaración Universal de la UNESCO sobre la Diversidad Cultural, Recuperado el 31 de marzo del 2006 de: hyyp://www.uasb.edu.edu.ec/padh/revista11/instrumentos/ declaración\%2ounesco.htm

UNICEF (2008). Visibilidad e invisibilidad de las culturas de los pueblos indígenas. Esterotipos y posible racismo en los libros de textos escolares. Bolivia, Ecuador y Perú. 